\title{
Whole-body magnetic resonance imaging (WB-MRI) with diffusion-weighted whole-body imaging with background body signal suppression (DWIBS) in prostate cancer: Prevalence and clinical significance of incidental findings
}

\section{Soma Kumasaka ( $\square$ kumasaka88@gunma-u.ac.jp )}

Gunma University Graduate School of Medicine School of Medicine: Gunma Daigaku Daigakuin Igakukei Kenkyuka Igakubu https://orcid.org/0000-0001-5541-3847

\section{Shunichi Motegi}

International University of Health and Welfare: Kokusai Iryo Fukushi Daigaku

\section{Yuka Kumasaka}

Gunma University Graduate School of Medicine School of Medicine: Gunma Daigaku Daigakuin Igakukei Kenkyuka Igakubu

\section{Tatsuya Nishikata}

Josai Clinic

Masami Otomo

Josai Clinic

\section{Yoshito Tsushima}

Gunma University Graduate School of Medicine School of Medicine: Gunma Daigaku Daigakuin Igakukei Kenkyuka Igakubu

\section{Research article}

Keywords: Prostate cancer, Whole-body MRI, DWIBS, Incidental findings

Posted Date: March 31st, 2021

DOl: https://doi.org/10.21203/rs.3.rs-362544/v1

License: (영 This work is licensed under a Creative Commons Attribution 4.0 International License. Read Full License

Version of Record: A version of this preprint was published at The British Journal of Radiology on June 16th, 2021. See the published version at https://doi.org/10.1259/bjr.20210459. 


\section{Abstract \\ Background}

Diffusion-weighted whole-body imaging with background body signal suppression (DWIBS) is now recommended as a first-line staging modality in prostate cancer patients, and the widespread use of DWIBS may lead to an increased frequency of incidental findings. The aim of this study is to evaluate the prevalence and clinical significance of incidental findings detected on whole-body magnetic resonance imaging (WBMRI) with DWIBS in patients with prostate cancer.

\section{Methods}

Data from 124 patients (age, $76.5 \pm 5.6$ years; range, 60-90) with pathologically confirmed prostate cancer, who underwent WB-MRI between December 2016 and April 2020, were retrospectively analyzed. Findings unrelated to prostate cancer were considered as incidental findings and categorized into two groups based on their clinical implications, as follow: imaging follow-up or additional examinations was required (significant incidental findings) and no need to additional work-up (non-significant incidental findings). A Chisquare test was performed to compare the differences in the prevalence of significant incidental findings based on age ( $\leq 75$ and $>75$ years old).

\section{Results}

A total of 334 incidental findings were found, with $8.1 \%(n=27)$ as significant incidental findings and $91.9 \%$ $(n=307)$ as non-significant incidental findings. Significant incidental findings were more frequent in patients over 75 years old than those of 75 years old or younger $(28.6 \%$ vs $11.1 \%, p=0.018)$. Nineteen of the 27 significant incidental findings $(70.4 \%)$ were observed on non-DWIBS sequences.

\section{Conclusion}

Clinically significant incidental findings, which required imaging follow-up or additional examinations, were commonly observed in patients with prostate cancer on WB-MRI/DWIBS.

\section{Background}

Recent advances in diagnostic imaging technologies have allowed us to obtain whole-body magnetic resonance imaging (WB-MRI), which provides high-quality anatomical images of the entire human body within reasonable examination times. Diffusion-weighted whole-body imaging with background body signal suppression (DWIBS) was reported as a unique concept for whole-body diffusion-weighted imaging in 2004 [1]. This technique provides high-quality positron emission tomography (PET)-like images without radiation exposure or contrast agent administration [2]. DWIBS is now considered a feasible clinical technique for evaluating primary and metastatic lesions in adult patients with high sensitivity and accuracy [3-7]. In 
particular, DWIBS is now recommended as a first-line diagnostic imaging for tumor staging in patients with prostate cancer [8]. However, the widespread use of DWIBS may lead to an increased frequency of incidental findings. Although some of these incidental findings may be beneficial to patients, for example in the case of earlier detection of second primary malignancy, they may lead to increased patient anxiety, radiation exposure, and healthcare costs due to additional examinations required [9-12].

Some studies have reported the prevalence of incidental findings in healthy volunteers on WB-MRI [13-15], but data regarding the prevalence of incidental findings on WB-MRI for cancer patients and its clinical impact remain limited.

The aim of this study was to clarify the prevalence of incidental findings detected on WB-MRI/DWIBS in patients with prostate cancer and their clinical significance.

\section{Methods}

\section{Patients}

All prostate cancer patients who underwent WB-MRI/DWIBS at our clinic between December 2016 and April 2020 were retrospectively analyzed. Inclusion criteria were: 1) pathologically confirmed prostate cancer; and 2) age $>20$ years old. Results from second or subsequent WB-MRI/DWIBS of the same patients were excluded from this study, and only data from the first WB-MRI/DWIBS were analyzed. Since the aim of this study was to assess the incidental findings on WB-MRI/DWIBS in clinical settings, scans with poor image quality due to patient-related factors (e.g., motion artifact, gibbus deformity) were NOT excluded from the analysis.

The institutional review board approved all study protocols. Due to the retrospective nature of the study, informed consent from each patient was waived, and the opt-out method on our hospital website was employed.

WB-MRI/DWIBS protocol

All WB-MRI/DWIBS studies were performed using a 1.5-T MRI scanner (Achieva; Philips Medical Systems, Best, the Netherlands) using a sliding surface coil approach [16]. No contrast agent was used. The WBMRI/DWIBS protocol was shown in Table 1. Maximum intensity projections (MIPs) of coronal and sagittal images and volumetric view in DWIBS images were reconstructed from axial planes. Fusion images of DWIBS and STIR in three orthogonal directions were also created. Total scan time was about $35 \mathrm{~min}$.

Imaging interpretation and classification of incidental findings

All WB-MRI/DWIBS images were retrospectively reviewed by two board-certified diagnostic radiologists for this study. If the findings detected on WB-MRI/DWIBS were considered unrelated to prostate cancer, they were considered as incidental. If incidental findings were identified, recommendations for management were documented. We categorized incidental findings into two groups based on clinical implication: nonsignificant, if the finding did not require further evaluation, imaging follow-up or treatment (e.g., old brain 
infarcts, renal or hepatic cysts, vertebral compression fracture without compression of nervous structures); significant, if the finding was considered to be required further evaluation to characterize findings, imaging follow-up or treatment (e.g., cystic lesions of the pancreas, pleural effusion, cardiomegaly, indeterminate masses or nodules in any organs). These significant incidental findings potentially lead to changes in treatment strategy of the patients. In cases of disagreement between two observers, a final consensus was reached though inter-observer discussion.

Statistical analyses

All statistical analyses were performed using SPSS software (IBM SPSS Statistics 25; IBM Japan, Tokyo, Japan). A Chi-square test was performed to compare the differences in the prevalence of significant incidental findings based on age ( $\leq 75$ and $>75$ years old). A $p$-value of $<0.05$ was considered significant.

\section{Results}

A total of 135 WB-MRI/DWIBS scans were performed for 124 consecutive prostate cancer patients during the study period. Eleven examinations were excluded as second scans $(n=9)$, third scans $(n=1)$, or fourth scans $(n=1)$, so 124 cases were included for further analyses (age, $76.5 \pm 5.6$ years; range, $60-90$ years).

All 124 patients showed at least one incidental finding on WB-MRI/DWIBS (Figs. 1-3). A total of 334 incidental findings were identified, with one incidental finding in 36 patients and two or more incidental findings in 88 patients. Of these findings, 27 lesions (8.1\%) in 26 patients $(21.0 \%)$ were considered as significant incidental findings, and further imaging $(n=21)$, echocardiogram and blood test $(n=3)$, urine and blood test $(n=1)$, endoscopy $(n=1)$, or nasopharyngolaryngoscopy $(n=1)$ were recommended. Of these 27 findings, six lesions in six patients $(4.8 \%)$ were considered highly suspicious for malignancy. Contrastenhanced computed tomography (CT) $(n=9)$ and magnetic resonance cholangiopancreatography (MRCP) ( $n$ $=8$ ) were the most frequent imaging modalities recommended for follow-up or further evaluation of significant incidental findings, followed by echocardiogram $(n=3)$, contrast-enhanced MRI $(n=2)$, and unenhanced CT $(n=2)$. The most common incidental findings recommended further work-up were cystic lesions of the pancreas $(n=8)$ (Table 2).

Of these 27 significant incidental findings, 19 lesions (70.4\%) were detected on T2-weighted image (T2WI), and eight lesions were detected on DWIBS. These findings were more frequently observed in patients over 75 years old than those of 75 years old or younger $(28.6 \%$ vs $11.1 \%, p=0.018)$. Of the six lesions suspected of malignancy, five lesions were found in patients over 75 years old.

The rest of 307 incidental findings $(91.9 \%)$ were considered as non-significant. The most common findings were renal cysts ( 75 patients, $60.5 \%$ ), hepatic cysts (48 patients, $38.7 \%$ ), and benign spinal canal stenosis (24 patients, $19.4 \%)$.

\section{Discussion}

In the current study, the majority of incidental findings on WB-MRI/DWIBS were considered as clinically nonsignificant. However, some incidental findings were clinically significant with higher prevalence in older 
patients, and these may have impacts leading to changes in treatment strategy. It should be emphasized that 19 of the 27 significant incidental findings (70.4\%) in 26 patients were found on axial T2WI. We reconfirmed that T2WI plays an important role when reviewing DWI, as a previous study mentioned [17]. Instead of focusing only on DWIBS, T2WI must also be carefully evaluated.

Some previous studies have evaluated incidental findings on WB-MRI $[13-15,18-20]$. The prevalence of significant incidental findings in our study (21.0\%) was similar to that in previous studies $(2.9-36.8 \%)$. Of those six previous studies, only three applied DWI/DWIBS in the study protocols, and none of them mentioned the sequences from which incidental findings were identified. In a previous study of WB-MRI with DWIBS in patients with prostate cancer, incidental findings were found in only $31 \%$ (31/100) of them [21]. This lower detection rate was probably due to the research focus, comparing DWIBS with bone scintigraphy and $\mathrm{CT}$ for the detection of bone metastasis. Actively searching for extraosseous lesions likely increase the detection rate of incidental findings.

In our study, only $1.6 \%$ of patients $(2 / 124)$ showed lung nodule or pulmonary hilar mass, although these findings were reported to be found in $3.8 \%(4 / 104)$ and $4.4 \%(15 / 344)$ of patients on whole-body CT [22, 23]. This is probably due to the limited role of MRI in the detection of lung nodules, given the lower spatial resolution compared to multidetector-row $\mathrm{CT}$ and the physiologic noise associated with cardiac and respiratory motions.

The issues regarding incidental findings are not only how often they occur, but also how much impact they have clinically and how to report them. Radiologists should play critical roles in deciding whether a detected lesion is benign or having potential clinical importance. In this study, we categorized the incidental findings into two groups based on the necessity of further work-up-trying to classify the findings according to the most common situations in daily practice. In our study, six patients (4.8\%) were considered as having lesions highly suggestive of second malignancy. This result was quite important, since these findings potentially lead to changes in treatment strategy of patients with prostate cancer. Furthermore, additional examinations such as CE-CT were required for further investigation of these findings, they can lead to increased healthcare costs and radiation exposure. Although WB-MRI/DWIBS can provide a comprehensive evaluation of whole-body in cancer patients-detecting metastasis not only in bone marrow, but also in lymph node or other organs-it is not an almighty technique, and additional work-up for evaluation of encountered incidental findings is inevitable. These kinds of suspected malignant lesions were also found in patients with other diseases such as lymphoma (4.4\%), neurofiblomatosis (1.6\%), and multiple myeloma $(2.9 \%)$ in previous studies with WBMRI [18-20]. Clinicians should inform patients of these possible incidental findings and the additional examination prior to WB-MRI/DWIBS as part of their informed consent process.

This study had several limitations. First, incidental findings in our study were diagnosed based on MRI features, in the absence of clinical or pathological confirmation. Second, we did not have access to clinical follow-up data for most patients, as all of our patients received primary care in other clinics or hospitals and visited the study site only for imaging consultations.

\section{Conclusion}


Our study revealed that clinically significant incidental findings were commonly observed on WB-MRI in patients with prostate cancer, requiring further examination. Checking the entire organ carefully -not only on DWIBS, but also on T2WI- for abnormalities is important. Radiologists should clearly and precisely report any incidental findings detected when reviewing WB-MRI, and clinicians should inform patients of the probability of incidental findings as part of their informed consent process.

\section{Declarations}

Ethics approval and consent to participate: Approved by Gunma University Ethical Review Board for Medical Research Involving Human Subjects. HS2020-054, 2020/07/17, retrospectively registered. Due to the retrospective nature of the study, informed consent was carried out using the opt-out method on our hospital website.

Consent to publish: Not applicable

Availability of data and material: The datasets used and/or analyzed during this study are available from the corresponding author on reasonable request.

Competing interests: The authors declare that they have no conflicts of interest.

Funding: None.

\section{Authors' contributions:}

SK: Project development, Data collection and management, Data analysis, Manuscript writing

SM: Protocol development, Manuscript editing

YK Data collection and management, Data analysis

TN: Protocol development, Clinical studies

MO: Protocol development, Clinical studies

YT: Manuscript editing

Acknowledgements: None.

\section{Authors' Information:}

Corresponding author: Soma Kumasaka

Department of Diagnostic Radiology and Nuclear Medicine

Gunma University Graduate School of Medicine

Maebashi, Gunma 371-8511, Japan 
Tel: 027-220-8401, Fax: 027-220-8409

Email address: kumasaka88@gunma-u.ac.jp

\section{References}

1. Takahara T, Imai Y, Yamashita T et al (2004) Diffusion weighted whole body imaging with background body signal suppression (DWIBS): technical improvement using free breathing, STIR and high resolution 3D display. Radiat Med 22:275-282

2. Kwee TC, Takahara T, Ochiai R et al (2008) Nievelstein RAJ, Luijten PR. Diffusion-weighted whole body imaging with background body signal suppression (DWIBS): features and potential applications in oncology. Eur Radiol 18:1937-1952

3. Nakanishi K, Kobayashi M, Nakaguchi K et al (2007) Whole-body MRI for detecting metastatic bone tumor: diagnostic value of diffusion-weighted images. Magn Reson Med Sci 6:147-155

4. Takenaka D, Ohno Y, Matsumoto K et al (2009) Detection of bone metastases in non-small cell lung cancer patients: comparison of whole-body diffusion-weighted imaging (DWI), whole-body MR imaging without and with DWI, whole-body FDG-PET/CT, and bone scintigraphy. J Magn Reson Imaging 30:298308

5. Laurent V, Trausch G, Bruot O et al (2010) Comparative study of two whole-body imaging techniques in the case of melanoma metastases: advantages of multi-contrast MRI examination including a diffusionweighted sequence in comparison with PET-CT. Eur J Radiol 75:376-383

6. Sakurai Y, Kawai H, Iwano S et al (2013) Supplemental value of diffusion-weighted whole-body imaging with background body signal suppression (DWIBS) technique to whole-body magnetic resonance imaging in detection of bone metastases from thyroid cancer. J Med Imaging Radiat Oncol 57:297-305

7. Stecco A, Romano G, Negru M et al (2009) Whole-body diffusion-weighted magnetic resonance imaging in the staging of oncological patients: comparison with positron emission tomography computed tomography (PET-CT) in a pilot study. Radiol Med 114:1-17

8. Lecouvet FE, Talbot JN, Messiou C et al (2014) Monitoring the response of bone metastases to treatment with Magnetic Resonance Imaging and nuclear medicine techniques: a review and position statement by the European Organisation for Research and Treatment of Cancer imaging group. Eur J Cancer 50:25192531

9. Pandharipande PV, Herts BR, Gore RM et al (2016) Rethinking Normal: Benefits and Risks of Not Reporting Harmless Incidental Findings. J Am Coll Radiol 13:764-767

10. Berland LL (2013) Overview of white papers of the ACR incidental findings committee ii on adnexal, vascular, splenic, nodal, gallbladder, and biliary findings. J Am Coll Radiol 10:672-674

11. Ding A, Eisenberg JD, Pandharipande PV (2011) The economic burden of incidentally detected findings. Radiol Clin North Am 49:257-265

12. Adams SJ, Babyn PS, Danilkewich A (2016) Toward a comprehensive management strategy for incidental findings in imaging. Can Fam Physician 62:541-543 
13. Morin SH, Cobbold JF, Lim AK et al (2009) Incidental findings in healthy control research subjects using whole-body MRI. Eur J Radiol 72:529-533

14. Hagenscheid K, Seipel R, Schmidt CO et al (2013) Potentially relevant incidental findings on research whole-body MRI in the general adult population: frequencies and management. Eur Radiol 23:816-826

15. Cieszanowski A, Maj E, Kulisiewicz P et al (2014) Non-contrast- enhanced whole-body magnetic resonance imaging in the general population: the incidence of abnormal findings in patients 50 years old and younger compared to older subjects. PLoS One 26:e107840

16. Takahara T, Kwee TC, Kibune S et al (2010) Whole-body MRI using a sliding table and repositioning surface coil approach. Eur Radiol 20:1366-1373

17. Tsushima Y, Takano A, Taketomi-Takahashi A et al (2007) Body Diffusion-Weighted MR Imaging Using High B-Value for Malignant Tumor Screening: Usefulness and Necessity of Referring to T2-weighted Images and Creating Fusion Images. Acad Radiol 14:643-650

18. Galia M, Albano D, Narese D et al (2016) Whole-body MRI in patients with lymphoma: collateral findings. Radiol Med 121:793-800

19. Jaremko JL, Macmahon PJ, Torriani M et al (2012) Whole body MRI in neurofibromatosis: incidental findings and prevalence of scoliosis. Skeletal Radiol 41:917-923

20. Wale A, Pawlyn C, Kaiser M et al (2016) Frequency, distribution and clinical management of incidental findings and extramedullary plasmacytomas in whole body diffusion weighted magnetic resonance imaging in patients with multiple myeloma. Haematologica 101:e142-144

21. Lecouvet FE, El Mouedden J, Collette L et al (2012) Can whole-body magnetic resonance imaging with diffusion-weighted imaging replace Tc $99 \mathrm{~m}$ bone scanning and computed tomography for single-step detection of metastases in patients with high-risk prostate cancer? Eur. Urol 62:68-75

22. Seah MK, Murphy CG, McDonald S et al (2016) Incidental findings on whole-body trauma computed tomography: experience at a major trauma centre. Injury 47:691-694

23. Liu W, Mortelé KJ, Silverman SG (2005) Incidental extraurinary findings at MDCT urography in patients with hematuria: prevalence and impact on imaging costs. AJR Am J Roentgenol 185:1051-1056

\section{Tables}


Table 1. MR sequences applied in the WB-MRI study

\begin{tabular}{|c|c|c|c|c|c|}
\hline Parameters & DWIBS & STIR TSE & $\begin{array}{l}\text { T1W } \\
\text { TSE }\end{array}$ & In/Out GRE & $\begin{array}{l}\text { T2W } \\
\text { TSE }\end{array}$ \\
\hline $\begin{array}{l}\text { Imaged } \\
\text { area }\end{array}$ & $\begin{array}{l}\text { neck- } \\
\text { pelvis }\end{array}$ & neck-pelvis & $\begin{array}{l}\text { neck- } \\
\text { pelvis }\end{array}$ & neck-pelvis & $\begin{array}{l}\text { neck- } \\
\text { pelvis }\end{array}$ \\
\hline Plane & axial & sagittal, coronal & sagittal & axial, sagittal, coronal & axial \\
\hline $\begin{array}{l}\mathrm{TR} / \mathrm{TE} \\
\text { (msec) }\end{array}$ & $8461 / 82$ & $2773 / 60$ & $642 / 12$ & $180-216 / 2.3 / 4.6$ & $3685 / 80$ \\
\hline $\begin{array}{l}\text { Flip angle } \\
\text { (deg) }\end{array}$ & & 90 & 90 & 75 & 90 \\
\hline FOV $(\mathrm{mm})$ & $370 \times 284$ & $330 \times 330 / 330 \times 411$ & $330 \times 302$ & $370 \times 289 / 330 \times 330 / 330 \times 409$ & $370 \times 296$ \\
\hline Matrix & $112 x$ & $224 x$ & $336 x$ & $256 / 256 / 272$ & $256 x$ \\
\hline $\begin{array}{l}\text { Slice } \\
\text { thickness } \\
(\mathrm{mm})\end{array}$ & 5.5 & $5.5 / 7.0$ & 5.5 & $5.5 / 7.0 / 5.5$ & 5.5 \\
\hline $\begin{array}{l}\text { Scan } \\
\text { technique }\end{array}$ & IR & IR & TSE & Dual-FFE & TSE \\
\hline $\begin{array}{l}\text { Fast } \\
\text { Imaging }\end{array}$ & $\begin{array}{l}\text { EPI- } \\
\text { single } \\
\text { shot }\end{array}$ & TSE-multi shot & $\begin{array}{l}\text { TSE- } \\
\text { multi } \\
\text { shot }\end{array}$ & - & $\begin{array}{l}\text { TSE- } \\
\text { multi } \\
\text { shot }\end{array}$ \\
\hline $\begin{array}{l}\text { Fat } \\
\text { suppression }\end{array}$ & STIR & STIR & - & - & - \\
\hline $\begin{array}{l}\text { b-factors } \\
\left(\mathrm{s} / \mathrm{mm}^{2}\right)\end{array}$ & $0 / 900$ & - & - & - & - \\
\hline NSA & 4 & $2 / 1$ & 2 & 1 & 1 \\
\hline $\begin{array}{l}\text { Number of } \\
\text { sections }\end{array}$ & 3 & 3 & 3 & 3 & 3 \\
\hline $\begin{array}{l}\text { acquisition } \\
\text { time }\end{array}$ & $\begin{array}{l}4 \mathrm{~min} \\
5 \mathrm{sec}\end{array}$ & $\begin{array}{c}1 \mathrm{~min} 57 \mathrm{sec} / 42 \mathrm{sec} \\
(\mathrm{BH} \times 3)\end{array}$ & $\begin{array}{l}1 \mathrm{~min} \\
56 \mathrm{sec}\end{array}$ & $35 \mathrm{sec} / 41 \mathrm{sec} / 41 \mathrm{sec}$ & $\begin{array}{c}59 \mathrm{sec} \\
(\mathrm{BH} \times 4)\end{array}$ \\
\hline
\end{tabular}


(one

section)

ıble 2. Significant incidental findings detected on WB-MRI

\begin{tabular}{llr}
\hline cidental findings & Recommendation & $\begin{array}{r}\text { number of the patients } \\
(\mathrm{n}=124)\end{array}$ \\
\hline ystic lesion of the pancreas & MRCP & $8(6.5 \%)$ \\
ardiac dilation and pleural & echocardiogram and blood & $3(2.4 \%)$ \\
fusion & test & \\
\hline sripancreatic nodule & CE-CT & $2(1.6 \%)$ \\
\hline arotid grand tumor & CE-MRI & $1(0.8 \%)$ \\
\hline esopharyngeal tumor* & nasopharyngolaryngoscopy & $1(0.8 \%)$ \\
\hline uymic tumor* & CE-CT & $1(0.8 \%)$ \\
\hline uymic cystic lesion & CE-MRI & $1(0.8 \%)$ \\
\hline llmonary hilar mass* & CE-CT & $1(0.8 \%)$ \\
\hline ing nodule* & CE-CT & $1(0.8 \%)$ \\
\hline eural mass* & CE-CT & $1(0.8 \%)$ \\
\hline astric-wall mass* & endoscopy & $1(0.8 \%)$ \\
\hline epatic tumor & CE-CT & $1(0.8 \%)$ \\
\hline nall adrenal nodule $(1 \mathrm{~cm})$ & Plain-CT & $1(0.8 \%)$ \\
\hline trenal mass $(2$ cm) & CE-CT & $1(0.8 \%)$ \\
\hline jelonephritis & urine and blood test & $1(0.8 \%)$ \\
\hline traperitoneal cystic mass & CE-CT & $1(0.8 \%)$ \\
\hline iravertebral mass & CE-CT & $1(0.8 \%)$ \\
\hline B & &
\end{tabular}

$\mathrm{B}$, whole-body; MRI, magagnetic resonance imaging; $\mathrm{MRCP}$, magnetic resonance ılangiopancreatography; CE, Contrast-enhanced; CT, computed tomography. iighly suspicious for malignancy 


\section{Figures}

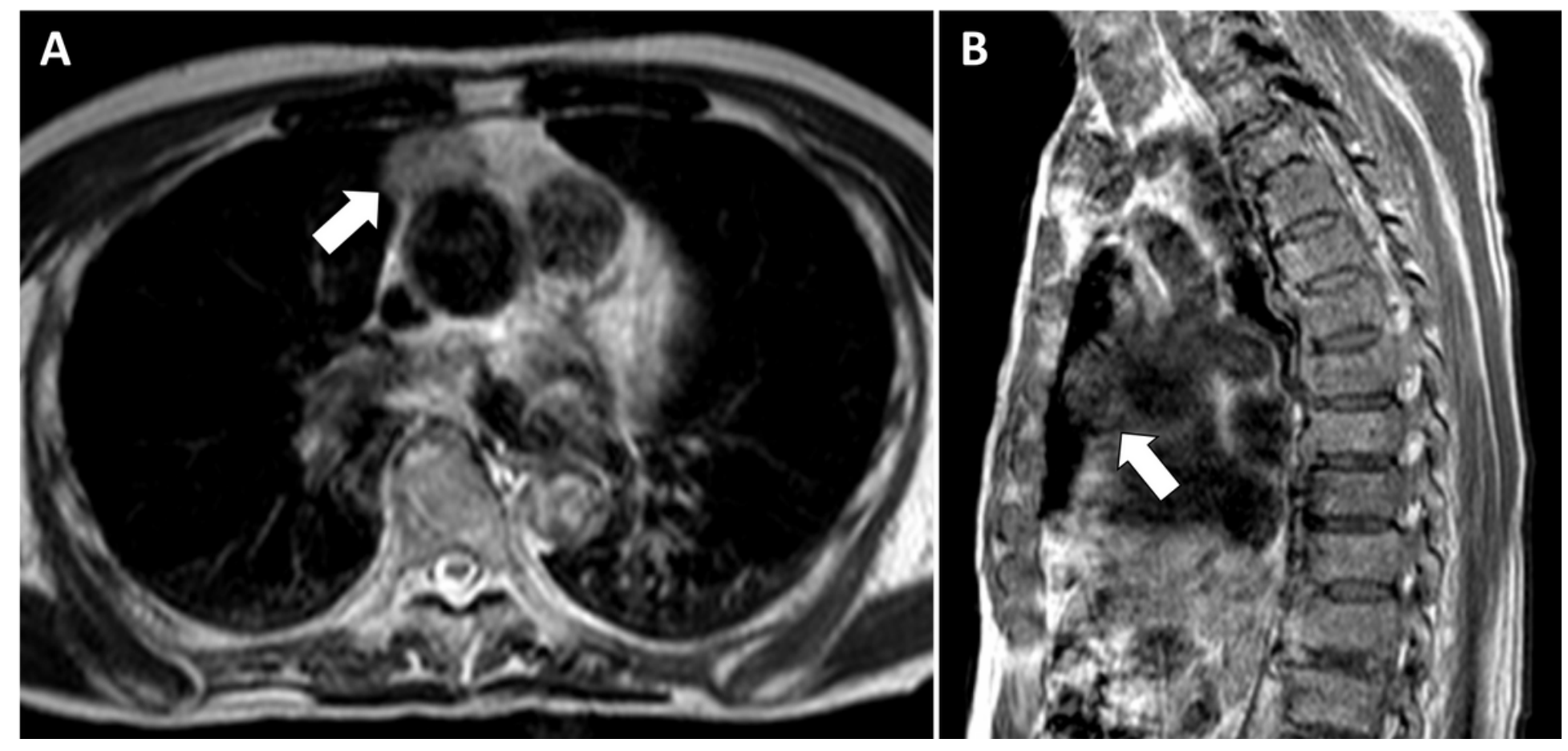

\section{Figure 1}

Thymic mass, categorized as a significant incidental finding in a patient in his 70s. Axial T2WI (a) and sagittal T1WI (b) show a mass in the anterior mediastinum (arrows). Contrast-enhanced computed tomography (CT) was recommended as further evaluation. 


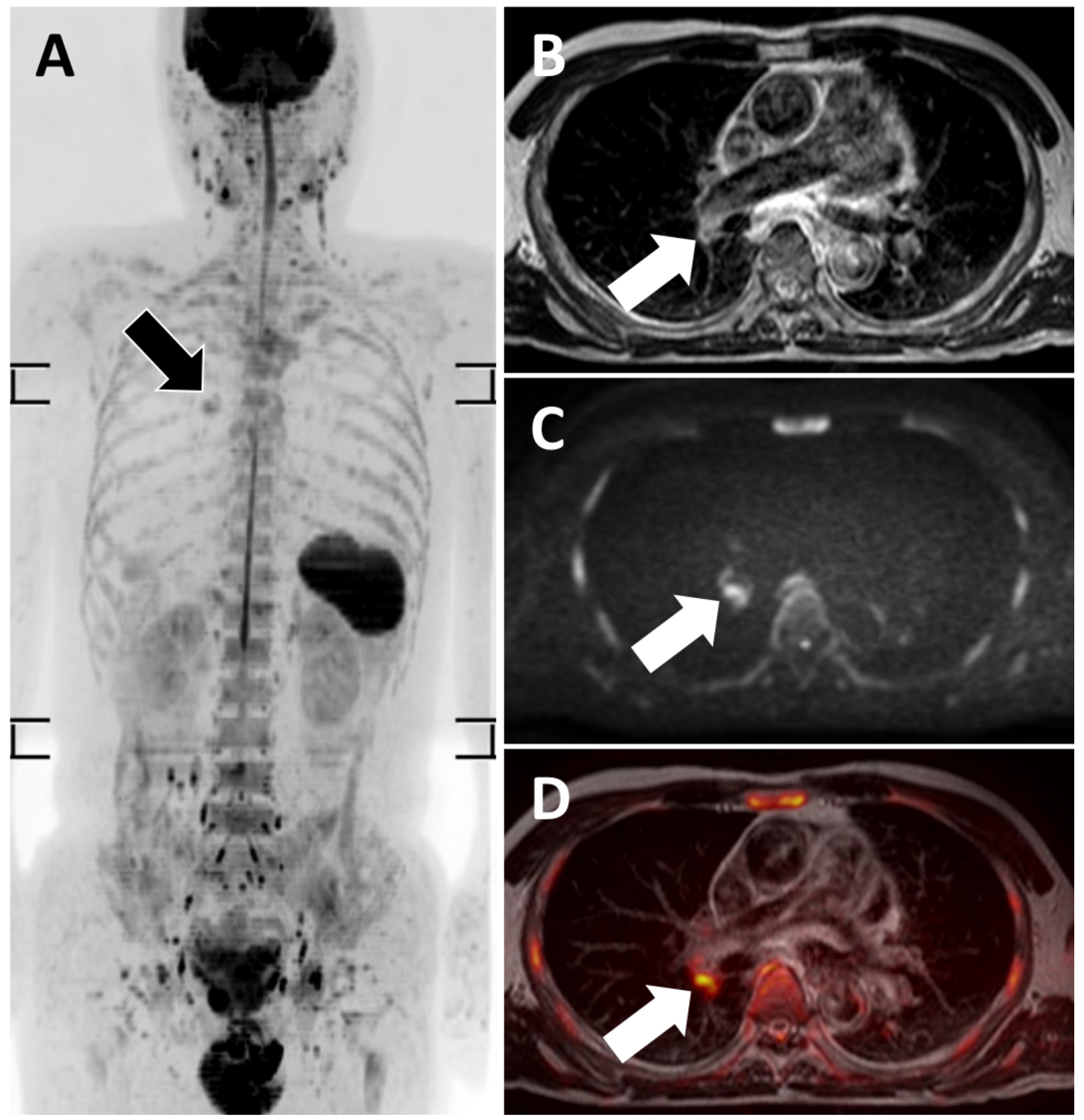

\section{Figure 2}

Right pulmonary hilar mass, categorized as a significant incidental finding in a patient in his 70s. DWIBS (a), axial T2WI (b), axial DWI (c), and axial DWI/T2WI fusion image (d) show a mass in the right pulmonary hilum (arrows). Contrast-enhanced CT was recommended as further evaluation. 


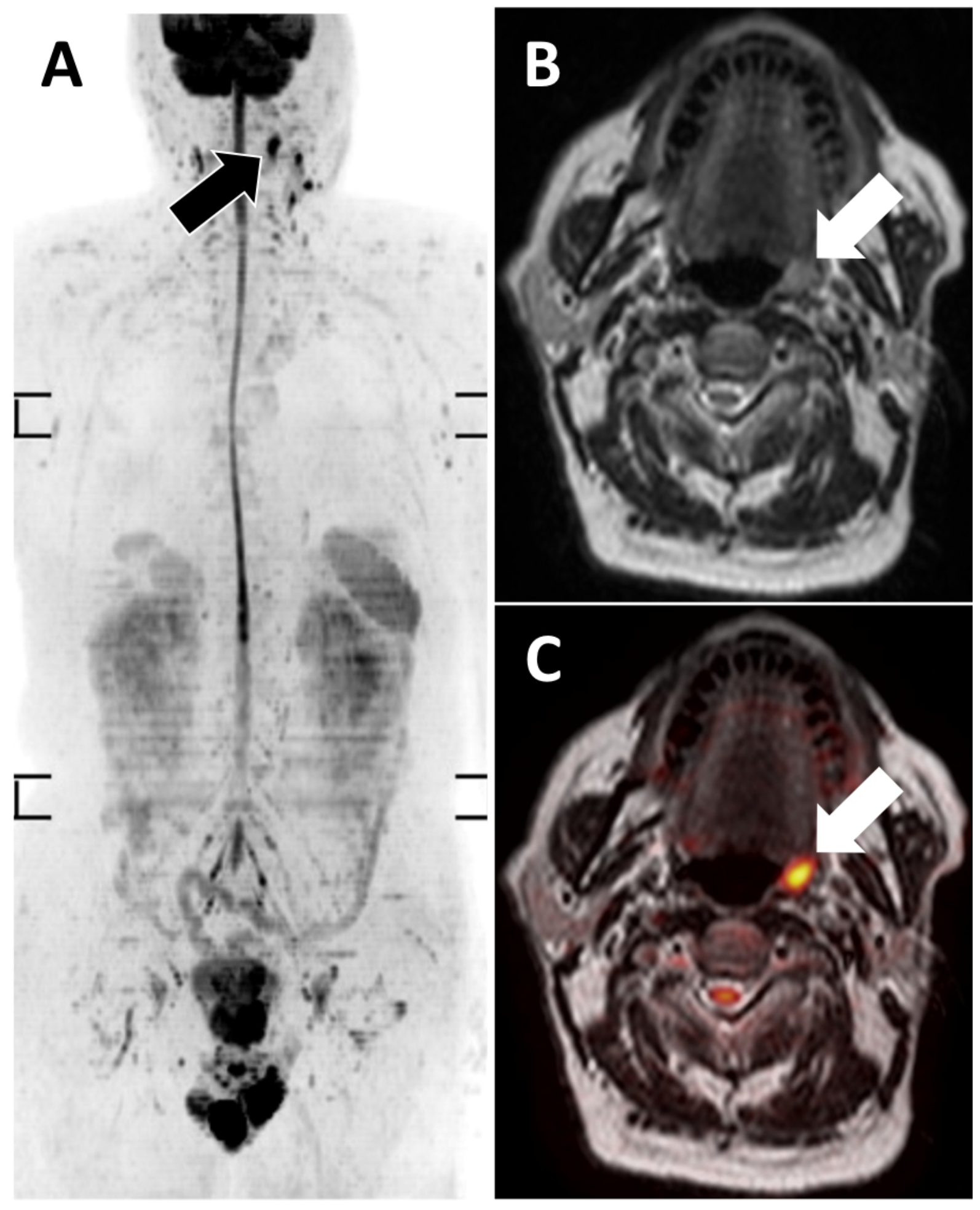

\section{Figure 3}

Mesopharyngeal mass, categorized as a significant incidental finding in a patient in his 70s. DWIBS (a), axial T2WI (b), and axial DWI/T2WI fusion image (c) show a mass in the left mesopharynx (arrows). Nasopharyngolaryngoscopy was recommended as further evaluation. 